\title{
EXPERIMENTAL VALIDATION OF THE BACKSCATTERING GAMMA-RAY SPECTRA WITH THE MONTE CARLO CODE
}

\author{
SY MINH TUAN HOANG ${ }^{\mathrm{a}, \mathrm{b}}$, SANGHO YOO ${ }^{\mathrm{a}}$, and GWANG MIN SUN ${ }^{\mathrm{a}, *}$ \\ ${ }^{a}$ Korea Atomic Energy Research Institute (KAERI) \\ PO Box 105, Yuseong, Daejeon 305-353, Korea \\ ${ }^{b}$ University of Science and Technology \\ 113 Gwahangno, Yuseong, Daejeon, Korea \\ *Corresponding author. E-mail : gmsun@kaeri.re.kr
}

Received December 21, 2010

In this study, simulations were done of a $661.6 \mathrm{keV}$ line from a point source of ${ }^{137} \mathrm{Cs}$ housed in a lead shield. When increasing the scattering angle from 60 to 120 degrees with a 6061 aluminum alloy target placed at angles of 30 and 45 degrees to the incident beam, the spectra showed that the single scattering component increases and that the multiple scattering component decreases. The investigation of the single and multiple scattering components was carried out using a MCNP5 simulation code. The component of the single Compton scattering photons is proportional to the target electron density at the point where the scattering occurs. The single scattering peak increases according to the thickness of the target and saturates at a certain thickness. The signal-to-noise ratio was found to decrease according to the target thickness. The simulation was experimentally validated by measurements. These results will be used to determine the best conditions under which this method can be applied to testing electron densities or to assess the thickness of samples to locate defects in them.

KEYWORDS : Gamma-ray spectra, Backscattering, MCNP, HPGe detector, NDT

\section{INTRODUCTION}

At present, non-destructive techniques (NDT) are well established in industrial, medical and security applications. Conventional NDTs such as transmission, radiographic and ultrasonic methods give quick results with high levels of accuracy. However, in many cases these methods are limited or not applicable if access to both sides of an object is restricted. In such a case, an alternative approach is a single-sided method in which the source and detector are arranged on the same side of the object [1]. This method is based on the measurement of Compton scattering photons[2].This process relies on measuring backscattering photons from the interior of an object surface when photons irradiate on the object.

Backscattering is a phenomenon in which photons colliding within the material are scattered backward compared to their original direction. When the material thickness is very thin, the backscattering phenomenon does not occur. The number of single and multiple backscattering photons increases according to the target thickness, eventually reaching saturation $[3,4]$. Based on the detailed structure of the High-Purity Germanium (HPGe) detector at the Neutron Activation Analysis Laboratory at the Korea Atomic Energy Research Institute (KAERI), we carried out a simulation of the photon spectrum of backscattering gamma rays from a 6061 aluminum alloy using a MCNP5 [5] simulation code. Based on this simulation, we investigated the spectrum of the photons-backscattered from the aluminum alloy by rotating the detector collimator from 60 to 120 degrees using $\mathrm{a}^{137} \mathrm{Cs}$ source at $661.6 \mathrm{keV}$ of energy to determine where the contribution of single scattering was greatest. We then fixed the scattering angle and changed the thickness of the aluminum target to find the saturated thickness of the aluminum alloy for single scattering.

\section{SIMULATION METHODS AND EXPERIMENS}

Monte Carlo based codes have proved to be a valuable tool in experimental design because they allow the testing of experimental conditions which would be difficult or expensive to perform otherwise. A number of Monte Carlo codes are available that simulate in detail photon and electron transport. Among these codes, we chose to use MCNP5 because it has been widely used in industry, medical and nuclear applications. It also has the advantage of allowing the simulation of an arbitrary geometry. In addition, MCNP5 has several preeminent features compared 
to other codes which are suitable to such applications. These features are discussed in the next session. MCNP5 is a general-purpose code that is used to simulate coupled neutron, photon and electron transport [5]. It can simulate radiation transport in any element, compound or mix of materials. The MCNP5's abilities include complex 3-D geometries, flexible physics models and the availability of extensive continuous-energy cross-section libraries derived from evaluated nuclear data files (ENDF). The attractiveness of MCNP5 is mainly associated with its user-friendly input file in which a generic configuration of materials can be created with the aid of user-defined cells and surfaces.

In this study, the experiment was carried out using backscattered geometry. $\mathrm{A}^{137} \mathrm{Cs}$ radioactive source with 10 $\mathrm{Ci}$ of activity emitting $661.6 \mathrm{keV}$ gamma rays was produced by a Nuclitec device (model R6030, serial 1944GP). This radioactive source was compressed in the form of pellets of $\mathrm{CsCl}$. An outer capsule of stainless steel with a diameter of $21.3 \mathrm{~mm}$ and a length of $26.4 \mathrm{~mm}$ contained a stainless steel inner capsule containing the pellets. This source was placed in the middle of a cylindrical cavity with a depth of $300 \mathrm{~mm}$ and a diameter of $36.8 \mathrm{~mm}$ fabricated in a lead container. The lead rectangular container with dimensions of $750 \mathrm{~mm}$ x $400 \mathrm{~mm}$ x $530 \mathrm{~mm}$ was especially prepared to enclose the source for biological safety and minimize background risk to the desired risk-free level.According to the findings of Hassan et al. [6] and Hussein [7] regarding source collimatorsused with the Compton scatter nondestructive testing method, the lead cylindrical collimator used in this study was designed to have two parts. The first collimator part had an outer diameter of $73.8 \mathrm{~mm}$ and a length of $20 \mathrm{~mm}$ with an internal diameter of $10 \mathrm{~mm}$. The second collimator part had an inner diameter of $5 \mathrm{~mm}$, an outer diameter of $80.80 \mathrm{~mm}$ and a length of $60 \mathrm{~mm}$ and was fitted to the first part. This collimator set was placed coaxially adjacent to the cylindrical cavity to obtain a narrow beam of $661.6 \mathrm{keV}$ gamma rays.

The distance from the scatter center to the surface of the source collimator was maintained at $313 \mathrm{~mm}$. The target in this study was a 6061 aluminum alloy slab with dimensions of $80 \mathrm{~mm} \times 80 \mathrm{~mm}$ and a thickness of $0.4 \mathrm{~cm}$. This this target was tilted at 30 and 45 degrees relative to the incident beam. The elemental composition of the 6061 aluminum alloy is given in Table 1 .

The backscattering gammaray was recorded by an HPGe detector which was properly collimated with lead shielding. The HPGe detector was rotated from 60 to 120 degrees compared to the incident gamma rays emanating from the source. The lead container was placed at a height of $720 \mathrm{~mm}$ from the floor of the laboratory room, and the target sample and the HPGe detector with lead shieldingwere set on the table. The experimental set was installed in the center of the room to avoid scattering from the wall as well as the floor and ceiling of the room. Figure 1 presents the experimental layout of this study.
A reverse-electrode closed-end coaxial Ortec $\mathrm{HPGe}$ detector was used (serial number 47-TN22404A). It included a Ge crystal cylinder that had an outer diameter of $59.8 \mathrm{~mm}$ and a length of $70.5 \mathrm{~mm}$. Inside the Ge crystal was a hole with adiameter of $8.9 \mathrm{~mm}$ anda depth of 66.7 $\mathrm{mm}$. The outer n-type contact layer was a lithium, and a boron layer formed the inner p-type contact layer. The detector was held in an aluminum cylinder with a thickness of $0.76 \mathrm{~mm}$. The rounded corners of the Ge crystal had a radius $8 \mathrm{~mm}$ as noted by the manufacturer. This HPGe detector had a resolution of $1.93 \mathrm{keV}$ (FWHM) at a 1.33 $\mathrm{MeV}^{60} \mathrm{Co}$ energy line. The relative efficiency of the HPGe detector was measured to be $45.8 \%(1.33 \mathrm{MeV})$ with a peak-to-Compton ratio of 68:1. The HPGe detector was properly shielded by the arrangement of lead bricks around

Table 1. Typical Composition of 6061 Aluminum alloy

\begin{tabular}{c|c}
\hline Component & Amount (wt.\%) \\
\hline Aluminum & Balance \\
\hline Magnesium & $0.8-1.2$ \\
\hline Silicon & $0.4-0.8$ \\
\hline Iron & Max. 0.7 \\
\hline Copper & $0.15-0.40$ \\
\hline Zinc & Max. 0.25 \\
\hline Titanium & Max. 0.15 \\
\hline Manganese & Max. 0.15 \\
\hline Chromium & $0.04-0.35$ \\
\hline Others & 0.05 \\
\hline
\end{tabular}

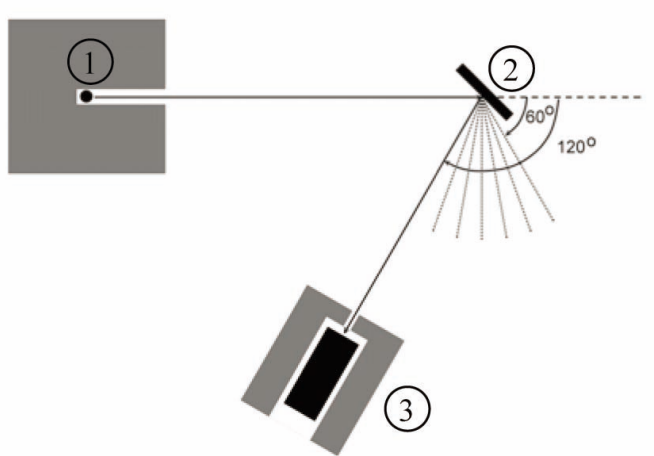

Fig. 1. The Experimental Layout of the Backscattering Effect Experiment:(1) Radioactive Source and Source Shielding, (2) Aluminum Alloy Sample, (3) the HPGe Detector and Detector Shielding 
it with a detector collimator in front facing the detector. To absorb the K X-rays emitted by the lead shielding, a copper plate was lined inside of the lead shielding. The detector collimator with an inner diameter of $5 \mathrm{~mm}$ was kept $180 \mathrm{~mm}$ away from the scatter center of the target. The measured data was recorded on a multi-channel analyzer (MCA), and the commercial program MAESTRO-32 was used to acquire and analyze the spectra for each measurement.

In the Monte Carlo codes, Visual Editor [8] and MCAM [9] were used to simplify the experimental geometry design, as these tools are robust for importing and converting the AutoCAD data to MCNP input text. We also applied the Worm code [10] to change the parameter of input file quickly when changing the geometry parameter setups of the angle of the detector rotations and the thickness of the aluminum alloy target.

To obtain the spectrum of the backscattering gamma with good statistics, a great many history runs are necessary (about $10^{11}$ particles) to overcome the low probability of scattering gamma rays in the direction of the detector. To reduce the computational time, we applied several variancereduction techniques in the MCNP simulation. The techniques known as surface source write/read (SSW/SSR), weight windows generation (WWG), energy cutoff (CUT) and source biasing [11] were applied. The SSW/SSR functions were used to write or read the surface source file for use in the subsequent MCNP simulation. This approach requires the use of a SSW/SSR card for proper recording and reading of the energies and trajectories of the incident gamma rays that cross the target, assuming that the gamma-ray energies and trajectories within the spatial distribution are nearly identical. With this approach, we can record the incident gamma raysat a virtual surface in front of the target, and the subsequent simulations are only performed by emitting the gamma ray from this surface with the help of a SSR card. The WWG makes it possible to generate important functions with respect to both the energy grid and the spatial grid that overlay the existing geometry [12]. The WWG can use considerable computational time to product a good result. As electron transport is very time-consuming, a considerable amount of computing time can be saved by setting a higher cutoff energy using the cut:e method. In these simulations, the energy cut off was set at $30 \mathrm{keV}$ for electron transport so as to decrease computing time. In the simulation, we used a collimator with a small inner diameter to obtain a narrow incident beam. Therefore, we used a direct beam with a 5 $\mathrm{mm}$ diameter in the MCNP simulation with a source-biasing technique. In addition, the coherent scatter was turned off when using the photon physics card (phys:p $2 \mathrm{j} 10$ ) to improve the F8 tally convergence. With these variancereduction techniques, the MCNP5 can run more than 2.1 billion histories. It integrates the useful features of variance reduction with pulse-height tallies (F8) and photon Doppler broadening, both of which were suitable for this simulation

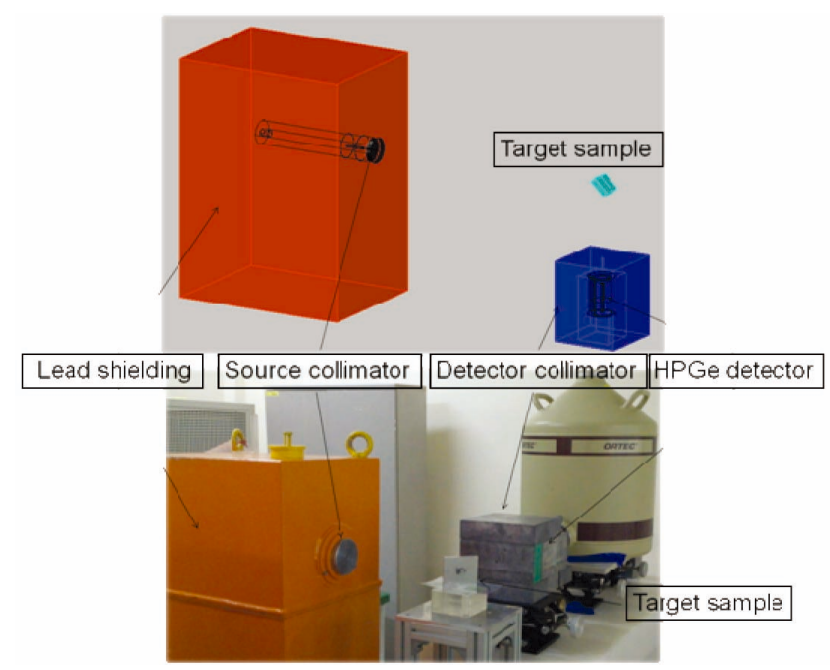

Fig. 2. The Modeling Figure and An Actual Experiment of the Backscattering Gamma-Ray Model

[14]. This running capability of greater than 2.1 billion histories is independent on the computer structure, whereas other codes such as MCNPX and GEANT4 are dependent on the hardware. Consequently, these variance reducing techniques and good integrating features served as a foundation for us to choose MCNP5 as the simulation tool in this study. The detector response tally F8 with the Gaussian energy broadening (GEB) option and the aluminum alloy material card with the composition shown in Table 1 were used in conjunction with the MCNP input deck.

In the measured spectra, the energy lines have a Gaussian shape. The MCNP5 code did not simulate this effect, instead using a technique to create an agreement in the peak width between the experiment and the calculation. The experimental value of the width, FWHM, was fitted with formula (1):

$$
\mathrm{FWHM}=\mathrm{a}+\mathrm{b}\left(\mathrm{E}+\mathrm{cE}^{2}\right)^{1 / 2}
$$

Here, $\mathrm{E}$ is the gamma-ray energy measured in $\mathrm{MeV}$; and $a, b$, and $c$ are parameters obtained from the fitness which can be used as MCNP5 input parameters. Once studying this detector configuration and using the energy peaks of a multi-nuclide point source for calibration, were obtained the results of $\mathrm{a}=0.00071, \mathrm{~b}=0.00075$, and $\mathrm{c}=$ 0.46493 . Figure 2 shows an experimental image and the related MCNP model.

\section{VALIDATION OF THE MCNP5 MODEL OF SCATTERING GAMMA-RAY SPECTRA}

To test the reliability of the MCNP5 geometry models, a comparison between the experimental and simulated efficiencies of these geometry models was carried out 
with a multi-nuclide standard source, as the efficiency strongly depends on the geometry in this system [13]. Therefore, this comparison was considered to be the best means of verifying the reliability of the geometry models in this work. Full energy peak efficiency calibration was carried out in the $60-1836 \mathrm{keV}$ energy range using a multi-nuclide standard source with radionuclides such as ${ }^{241} \mathrm{Am},{ }^{109} \mathrm{Cd},{ }^{57} \mathrm{Co},{ }^{123 \mathrm{~m}} \mathrm{Te},{ }^{51} \mathrm{Cr},{ }^{113} \mathrm{Sn},{ }^{85} \mathrm{Sr},{ }^{137} \mathrm{Cs},{ }^{88} \mathrm{Y}$ and ${ }^{60} \mathrm{Co}$. According to Sood et al.[14], the photon Doppler broadening must be taken into account for an accurate simulation of the low-energy photon transport. Usage of this photon Doppler broadening physics feature was controlled by the fifth entry of the phys:p card with a zero entry. In addition, the findings of Rodenas et al. [15] and Huy et al. [16] showed that the good agreement between the simulated and measured efficiencies can be obtained by adjusting the inactive Ge layer. We increased the inactive layer thickness several times for matching between the simulated and measured values at an accepted level. The result indicated that there was a good agreement between the calculated and measured efficiencies, within $6 \%$.

A second validation of the MCNP5 model for scattering gamma ray spectra involved a comparison between a simulated and measured spectrum. An intensely collimated gamma-ray beam from $\mathrm{a}^{137} \mathrm{Cs}$ radioactive source was made to impinge on a square target of an aluminum alloy with a thickness of $20 \mathrm{~mm}$. The target was fixed at 45 degrees relative to the incident beam and the scattered angle between the incident beam and the HPGe detector was 90 degrees. The typical simulated and measured spectrums are given in Figure 3. Because the angular aperture of the source collimator was very small and the diameter of the lead shielding was also small, the single scattering gamma ray from the target

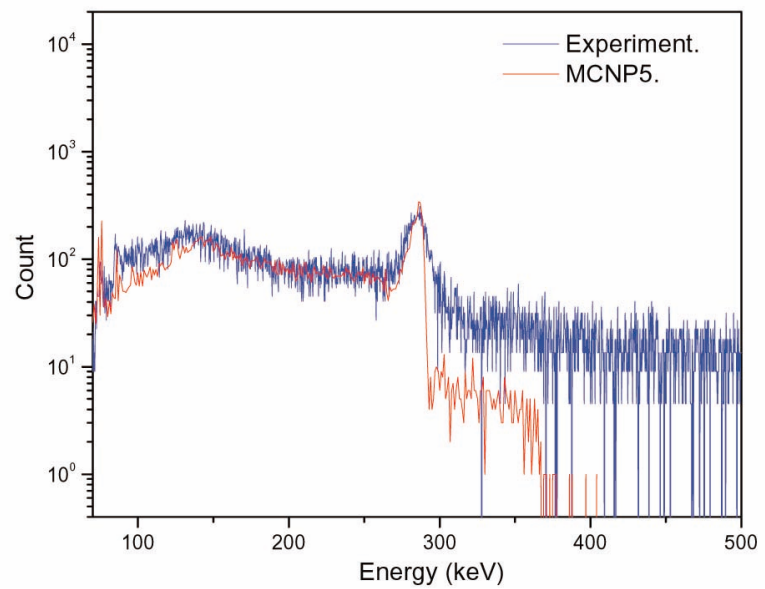

Fig. 3. The Simulated and Measured Result of the Scattered Spectrum for $661.6 \mathrm{keV}$ Gamma Rays at a Scattering Angle of 90 Degrees followed by another instance of scattering from the detector shielding had energy that was lower than that corresponding to the energy of the incoherent scattered peak. In Figure 3, the simulated and measured spectra are well matched. These spectra are a composite of single and multiple scattered gamma rays along with the background events. The energy of single scattering gamma rays in these spectra at a 90 degree scattering angle is approximately $288 \mathrm{keV}$ for $661.6 \mathrm{keV}$ incident gamma rays. In the Compton continuum of these spectra, a pair of two close-spaced phenomenal peaks can be observed. This is characteristic $\mathrm{K} \mathrm{X}$-ray doublet $\left(\mathrm{Bi}-\mathrm{K}_{\alpha}=77.107 \mathrm{keV}\right.$ and $\mathrm{Bi}-\mathrm{K}_{\beta 1}=87.349 \mathrm{keV}, \mathrm{Bi}-\mathrm{K}_{\beta 2}=89.784 \mathrm{keV}$ ) peaks originating from the lead shielding of the radioactive source and the HPGe detector, which are also matched between the two spectra. We also observed a double scattered peak from these spectra. Their positions at an energy of about $118 \mathrm{keV}$ are in good agreement with

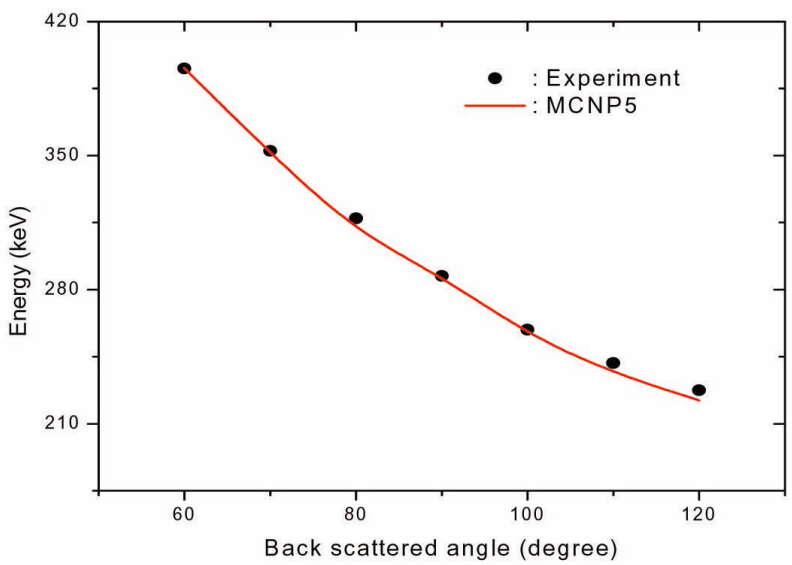

(a)

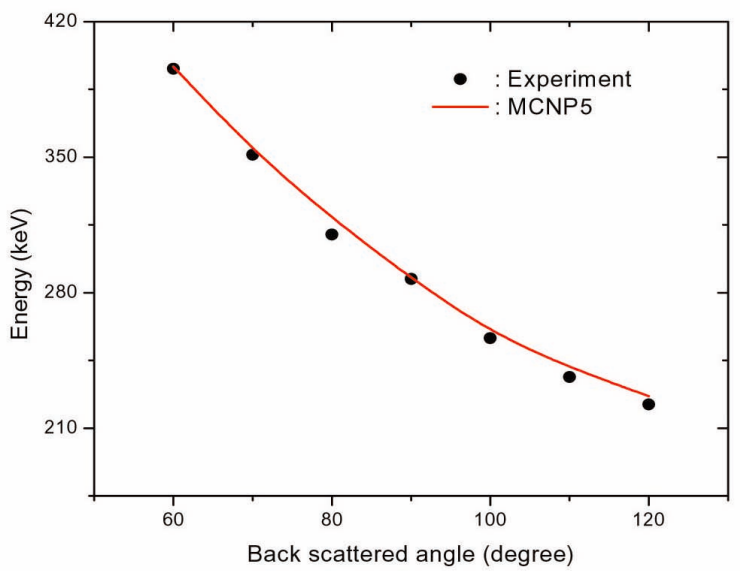

(b)

Fig. 4. The Dependence of the Single Scattering Peak with Backscattering Angles of (a) 30 Degrees, (b) 45 Degrees 
Equation 2, as provided by Fernandez [17], given below:

$$
\mathrm{E}=\frac{\mathrm{E}_{0} \mathrm{E}^{\prime}}{2 \mathrm{E}_{0}+\mathrm{E}^{\prime}}
$$

These validated results provide sufficient justification regarding the use of the MCNP5 model to simulate the spectrum for backscattering gamma rays in this experimental system.

\section{RESULTS AND DISCUSSION}

\subsection{Investigation of the Backscattering Gamma Rays with An Aluminum Alloy Target Placed at 30 and 40 Degrees}

An investigation of the spectrum of backscattering gammas rays while changing the angle between the radioactive source and the HPGe detector, $\theta$, was performed in the first set of simulations. At this stage, an aluminum alloy target with a thickness of $20 \mathrm{~mm}$ was placed at 30 and 40 degrees relative to the incident beam. When the HPGe detector with lead shielding was rotated by changing $\theta$ from 60 to 120 degrees, the backscattering peak shifted to a lower energy. This agrees with Compton scattering equation 3. Table 2 presents a comparison of the single scattering peak with the backscattered angle ranging from 60 to 120 degrees. Figure 4 shows the dependence of the single scattering peak with an aluminum alloy at 30 and 40 degrees compared to the incident beam.

$$
\mathrm{E}_{\gamma^{\prime}}=\frac{\mathrm{E}_{\gamma}}{1+\frac{\mathrm{E}_{\gamma}}{m_{e} c^{2}}(1-\cos \theta)}
$$

Here, $\mathrm{E}_{\gamma}$ and $\mathrm{E}_{\gamma}$ are incident and scattered energy, and $\theta$ is the backscattering angle.
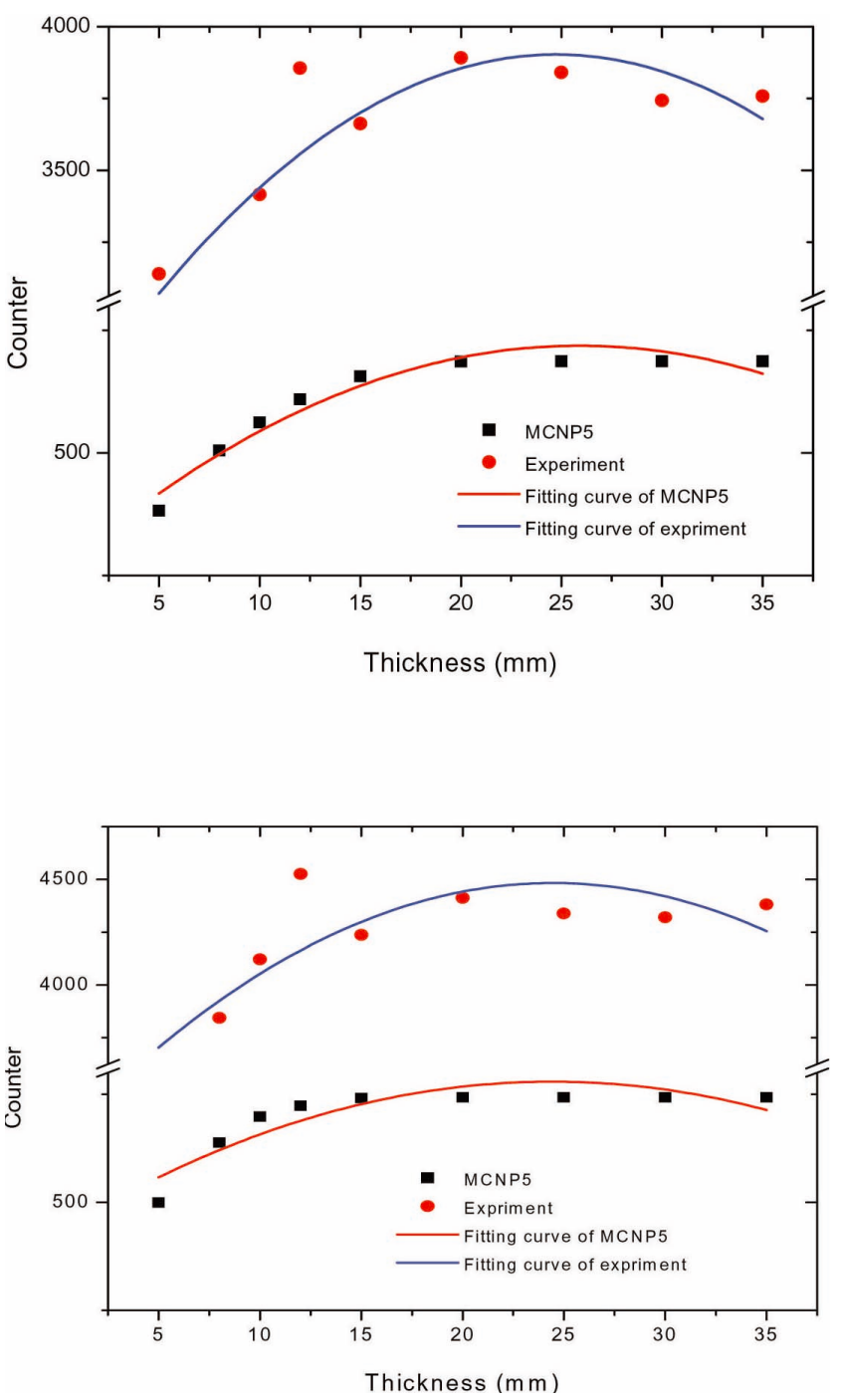

Fig. 5. Observed Variation of the Number of Singly Scattered Events Versus the Thickness of the Target at (a) 30 Degrees and (b) 45 Degrees

Table 2. A comparison of a single scattering peak with a backscattered angle ranging from 60 to 120 degrees

\begin{tabular}{c|c|c|c|c|c|c|c}
\hline \multirow{2}{*}{$\begin{array}{c}\text { Back- } \\
\text { scattered } \\
\text { angle } \\
\text { (degree) }\end{array}$} & \multirow{2}{*}{ Theoretical } & \multicolumn{3}{|c|}{30 degrees } & \multicolumn{3}{c}{40 degrees } \\
\cline { 3 - 8 } & & $\begin{array}{c}\text { Simulation } \\
(\mathrm{keV})\end{array}$ & $\begin{array}{c}\text { Experiment } \\
(\mathrm{keV})\end{array}$ & $\begin{array}{c}\text { Difference } \\
(\%)\end{array}$ & $\begin{array}{c}\text { Simulation } \\
(\mathrm{keV})\end{array}$ & $\begin{array}{c}\text { Experiment } \\
(\mathrm{keV})\end{array}$ & $\begin{array}{c}\text { Difference } \\
(\%)\end{array}$ \\
\hline 60 & 401.61 & 396 & 395.51 & 0.03 & 401 & 396.8 & 1.17 \\
\hline 70 & 357.26 & 351 & 352.43 & 0.35 & 357 & 353.46 & 1.03 \\
\hline 80 & 319.63 & 310 & 317.24 & 2.33 & 319 & 318.37 & 0.32 \\
\hline 90 & 288.31 & 287 & 287.14 & 0.05 & 288 & 287.4 & 0.21 \\
\hline 100 & 262.59 & 256 & 259.01 & 1.01 & 263 & 259.53 & 1.15 \\
\hline 110 & 241.68 & 236 & 241.72 & 2.27 & 242 & 241.2 & 0.17 \\
\hline 120 & 224.88 & 222 & 227.52 & 2.43 & 225 & 226.56 & 0.76 \\
\hline
\end{tabular}


The intensity of the backscattering peak increases while that of the multiple backscattering peak decreases according to the backscattering angle, which simplified the analysis of the spectra of the backscattering gamma rays when the backscattering angle had a relatively large value. We also achieved the location at which the contribution of single scattering was the largest.

\subsection{Investigation of Backscattering Gamma Rays According to the Thickness of the Aluminum Alloy}

In this case, we changed the thickness of the aluminum target from $0.4 \mathrm{~cm}$ to $2 \mathrm{~cm}$ when the HPGe detector was fixed at 90 degrees compared to the incident gamma rays emanating from the source. Figure 5 shows the dependence of the single scattering peak on the aluminum alloy thickness placed at 30 and 40 degrees. The result shows that the intensity of single and multiple backscattering photons increases according to the increase in the target thickness. However, the intensity of the backscatteredphotons increases up to a thickness of $20 \mathrm{~mm}$. We consider a thickness of $20 \mathrm{~mm}$ as the saturation depth of the backscattering inside the aluminum alloy target for the given energy and backscattering angle.

This is explained by the fact that gamma rays have a higher probability of scattering, while a backscatteredgamma ray must fly a longer distance before escaping the target surface when the gammaray penetrates at a deeper distance into the target. Hence, the absorbing and scattering process offset each other. Finally, a stage is reached when the thickness of the aluminum target becomes sufficient to counter balance two these processes, resulting in the reaching of the saturation depth.

\section{CONCLUSION}

In this study, a Monte Carlo simulation of backscattered gamma rays was carried out using MCNP5. The simulation and experiment were conducted at $661.6 \mathrm{keV}$ from ${ }^{137} \mathrm{Cs}$ radioactive source. The ${ }^{137} \mathrm{Cs}$ radioactive source was placed inside alead collimator and analuminum alloy target was placed at an angle of 30 and 40 degrees relative to the incident beam. The properties of backscattering gamma rays were then investigated while changing the thickness of the aluminum target and the scattering angle. The results showed that the single backscattering photons increase-while the multiple backscattering photons decrease according to the scattering angle relative to the incident beam. When the aluminum thickness was increased, the single and multiple backscattering photons increased and reached saturation values at a thickness of approximately $20 \mathrm{~mm}$. These results will be utilized to obtain the optimized condition with which this method can be applied to an investigation of electron densities or the thickness of samples to determine whether defects are present.

\section{ACKNOWLEDGEMENTS}

The authors would like to thank KAERI Radiological calibration Laboratory for supplying us with the Gamma Irraddiators (OB40)

\section{REFERENCES}

[ 1 ] L.M.N. Tavora, W.B. Gilboy, Study of Compton scattering signals in single-sided imaging applications using Monte Carlo methods, Nuclear Instruments and Methods in Physics Research B213 (2004) 155-161.

[2] Glenn F. Knoll, Radiation Detection and Measurement, John Wiley \& Sons, Inc (2000).

[3 ] Gurvinderjit Singh, Manpreet Singh, B.S. Sandhu, Bhajan Singh, Experimental investigation of multiple scattering of $662 \mathrm{keV}$ gamma rays in zinc at $90^{\circ}$, Radiation Physics and Chemistry 76 (2007) 750-758.

[4] Manpreet Singh, Gurvinderjit Singh, B.S. Sandhu, Bhajan Singh, Effect of detector collimator and sample thickness on $0.662 \mathrm{MeV}$ multiply Compton-scattered gamma rays, Applied Radiation and Isotopes 64 (2006) 373-378.

[5] X-5 Monte Carlo Team, MCNP - A General Monte Carlo N-Particle Transport Code, Version 5, Volume II: User's Guide. Los Alamos National Laboratory Report LA-CP03-0245, 2003.

[6] Hassan A. Jama, Esam M.A. Hussein, Design aspects of a gamma-ray energy-spectral Compton-scatter nondestructive testing method, Applied Radiation and Isotopes 50 (1999) 3731-342.

[7] Esam M.A. Hussein, Handbook on Radiation Probing, Gauging, Imaging and Analysis, Volume II, Kluwer Academic Publishers (2004).

[ 8 ] http://www.mcnpvised.com/

[9] Y. Wua, FDS Team, CAD-based interface programs for fusion neutron transport simulation, Fusion Engineering and Design 84 (2009) 1987-1992.

[10] http://worm.csirc.net/index.htm

[11] James Laird, Darby S. Kimball, MCNP Variance reduction techniques: what to use when and how, American nuclear society winter meeting (2009).

[12] Richard H. Olsher, A practical look at Monte Carlo variance reduction methods in radiations shielding, Nuclear Engineering and Technology, vol.38, No.3 April 2006.

[13] Jonas Boson, GoranAgren, Lennart Johansson, A detailed investigation of HPGe detector response for improved Monte Carlo efficiency calculations, Nucl. Instr. Meth., A 587 (2008) 304-314.

[14] Avneet Sood and Morgan C. White, Doppler Energy Broadening for Incoherent Scattering in MCNP5, Part II, Los Alamos National Laboratory Report LA-UR 04-0488.

[15] J. Rodenas, A. Pascual, I. Zarza, V. Serradell, J. Ortiz, L. Ballesteros, Analysis of the influence of germanium dead layer on detector calibration simulation for environmental radioactive samples using the Monte Carlo method, Nucl. Instr. and Meth, A 496 (2003) 390-399.

[16] Huy N.Q., Binh D.Q., An V.X. (2007), "Study on the increase of inactive germanium layer in a high purity germanium detector after a long time operation applying MCNP code", Nucl. Instrum. Methods, A 573 384-388.

[17] Fernandez, J.E., Compton and Rayleigh double scattering of unpolarized radiation, Phys. Rev. (1991) A44, 4232-4248. 